\title{
Erratum to: Abstracts of Original Contributions ASNC2015 The 20th Annual Scientific Session of the American Society of Nuclear Cardiology
} September 17-20, 2015 Washington, DC

\section{ERRATUM TO: J NUCL CARDIOL VOLUME 22, NUMBER 4;744-81 \\ DOI $10.1007 /$ S12350-015-0205-5}

In abstract 301-18 from the ASNC 2015 Annual Scientific Sessions, one author was listed incorrectly in the July/August 2015 issue (J Nucl Cardiol 2015;22:763). R D Russell should have been listed as R Folks. Please see the corrected abstract below in its entirety:

\section{1-18}

INTER-OBSERVER REPRODUCIBILITY OF IDENTIFYING THE OPTIMAL CRT LEFT VENTRICULAR LEAD POSITION FROM SPECT MPI W ZHOU, ${ }^{1}$ Y ZHOU, ${ }^{2}$ R FOLKS, ${ }^{1}$ C D COOKE, ${ }^{1}$ $\mathrm{X}$ LIN,${ }^{3} \mathrm{~J} \mathrm{CHEN}^{1}{ }^{1}$ E V GARCIA ${ }^{1}$

${ }^{1}$ Emory University, Atlanta, GA, ${ }^{2}$ Nanjing Medical University, Nanjing, China, ${ }^{3}$ Anhui Medical University, Hefei, China

Background: A shift of the left ventricular (LV) lead position by $\sim 20 \mathrm{~mm}$ could impact on CRT response (PMID: 15173718). It is important that LV lead is placed away from scar and at or near the site of the latest activation. SPECT MPI has been shown to assess both LV mechanical dyssynchrony and viability to identify optimal LV lead position. This study was aimed to evaluate the reproducibility of identifying the optimal LV lead position from SPECT MPI.

Methods: The method was evaluated in 41 patients who underwent ECG-gated SPECT MPI. Two independent operators performed the following steps for each patient: (1) manually reorienting SPECT MPI transaxial slices into short-axis images; $* 2$ ) manually specifying the parameters characterizing LV location and size, such as LV center and radius, apex and base slices. Then, the short-axis images were submitted to an automatic sampling algorithm, which searched in 3D for maximal count circumferential profiles of LV. Both perfusion and phase polar maps were then generated to assess viability and dyssynchrony. On the perfusion polar map, the regions below $50 \%$ of the maximum perfusion were defined as scar regions. The EP 13-segment model was used to divide the polar maps for the optimal LV lead position measurement. The apical segments, septal segments, and the segments with more than 50\% scar were excluded. Amongst the remaining viable segments, the one with the latest activation was chosen as the optimal segment.

Results: In all the 41 patients, the optimal LV lead positions were identified by the two independent operators in the same segments in 29 patients, and in the immediate adjacent segments in 8 patients. In total, an agreement rate of $90.2 \%$ was achieved.

Conclusion: The preliminary results with 41 patients show that there is high inter-observer reproducibility when using our software to identify the optimal CRT LV lead position from SPECT MPI.

Acknowledgement: This study was supported by an AHA Grant (15POST22690035, PI: Weihua Zhou, $\mathrm{PhD})$.
The online version of the original article can be found under doi:10.1007/s12350-015-0205-5.

J Nucl Cardiol 2015;22:1316.

$1071-3581 / \$ 34.00$

Copyright (c) 2015 American Society of Nuclear Cardiology.

doi:10.1007/s12350-015-0305-2 